\title{
APLICAÇÃO DO MÉTODO DE IDENTIFICAÇÃO ESPECTRAL PARA IMAGENS DO SENSOR ASTER EM AMBIENTE DE CERRADO
}

\author{
Osmar Abílio de Carvalho Júnior ${ }^{1}$, Renato Fontes Guimarães², Éder de Souza Martins ${ }^{3}$, \\ Ana Paula Ferreira de Carvalho ${ }^{4}$ e Roberto Arnaldo Trancoso Gomes ${ }^{5}$
}

Recebido em 16 março, 2005 / Aceito em 10 outubro, 2005

Received on March 16, 2005 / Accepted on October 10, 2005

\begin{abstract}
The spectral classifiers allow a good estimate for the mapping of the materials from the similarity between the reference curve and the image. Initially the spectral classifiers had been developed for hyperspectral images analysis. However, some works demonstrate good results for the application of these techniques in multispectral images. The present work aims to evaluate the spectral classifier Spectral Identification Method (SIM) in ASTER image. The Spectral Identification Method (SIM) is proposed to establish a new similarity index and three estimates according to the significance levels ( $5 \%, 10 \%$ and $15 \%$ ) of the materials. This method is based on two statistical procedures: ANOVA and Spectral Correlation Mapper (SCM) coefficient. This information can be used to evaluate the degree of correlation among the materials in analysis. The advantage of this method is to validate according to levels of significance of the most probable areas of the sought material. The method was applied to ASTER image at the Military Instruction Field located Formosa (GO) close to Federal District. The images were acquired with atmosphere correction. The pixels size from the SWIR image were duplicated in order to join the VNIR and SWIR images. Endmembers were detected in three steps: a) spectral reduction by the Minimum Noise Fraction (MNF) transformation, b) spatial reduction by the Pixel Purity Index (PPI) and c) manual identification of the endmembers using the $\mathrm{N}$-dimensional visualizer. The classification was made from the endmembers of nonphotosynthetic vegetation (NPV), photosynthetic vegetation (PV) and soil. These procedures allowed to identify the main scenarios in the study area.
\end{abstract}

Keywords: remote sensing, image processing, spectral classifier.

RESUMO. As técnicas de análise espectral permitem uma boa estimativa para a identificação do material procurado pela similaridade entre a curva de referência e a da imagem. Inicialmente os classificadores espectrais foram desenvolvidos para análise de imagens de sensores hiperespectrais. No entanto, vários trabalhos vêm demonstrando bons resultados para a aplicação dessas técnicas em imagens multiespectrais. 0 presente trabalho possui como objetivo testar 0 classificador espectral denominado Método de Identificação Espectral (MIE) para imagens multiespectrais do sensor ASTER. 0 MIE fornece um novo índice de similaridade e três estimativas segundo níveis de significância das possíveis áreas do material procurado. Esse método fundamenta-se no procedimento estatístico ANOVA e no classificador espectral Spectral Correlation Mapper (SCM). Essa informação pode ser utilizada para avaliar o grau de correlação dos materiais em análise, sendo útil em uma análise exploratória. A vantagem desse método é que permite validar estatisticamente a existência do elemento procurado conforme um nível de significância. 0 método foi aplicado na imagem ASTER referente à área do campo de instrução militar de formosa situada no município de Formosa (G0) próxima ao Distrito Federal. As imagens utilizadas (VNIR e SWIR) foram adquiridas já corrigidas do efeito atmosférico. Duplicando o tamanho dos pixels da imagem SWIR foi feita a união entre as resoluções espaciais entre as imagens VNIR e SWIR. Os membros finais da área de estudo foram detectados através da seguinte seqüência: redução espectral pela transformação MNF (Minimum Noise Fraction), redução espacial pelo índice de pureza de pixel (PPI) e pelo visualizador n-dimensional. A partir dos membros finais referentes à vegetação fotossinteticamente ativa, vegetação não fotossiteticamente ativa e solo realizou-se a classificação. Esta metodologia permitiu identificar os principais cenários na área de estudo.

Palavras-chave: sensoriamento remoto, processamento de imagem, classificador espectral.

1 Universidade de Brasília, Departamento de Geografia, Campus Universitário Darcy Ribeiro, Asa Norte, 70910-900 Brasília, DF, Brasil. Fone: (61) 3307-2373; Fax: (61) 3272-1909 - E-mail: osmarjr@unb.br

2Universidade de Brasília, Departamento de Geografia, Campus Universitário Darcy Ribeiro, Asa Norte, 70910-900 Brasília, DF, Brasil. Fone: (61) 3307-2373; Fax: (61) 3272-1909 - E-mail: renatofg@unb.br

3 EMBRAPA Cerrados - Rodovia Brasília Fortaleza, km 18 Planaltina Distrito Federal. Fone: (61) 3388-9870 - E-mail: eder@cpac.embrapa.br

${ }^{4}$ Universidade de Brasília, Departamento de Ecologia, Campus Universitário Darcy Ribeiro - ICC Centro, 70910-900 Brasília, DF, Brasil. Fone: (61) 3367-5001; Fax: (61) 3272-1909-E-mail: anapaula@unb.br

${ }^{5}$ Departamento de Geografia, Universidade Federal do Rio de Janeiro (UFRJ) - E-mail: beto@igeo.ufrj.br 


\section{INTRODUÇ̃̃̃O}

0 advento da espectrometria imageada possibilitou um avanço nas técnicas de análise espectral de imagens. Muitas dessas técnicas vêm sendo adotadas no processamento de imagens multitemporais com bastante êxito (Adams et al., 1995; Rowan \& Mars, 2003; Sabol et al., 2002). Dentro desse contexto, o Método de Identificação Espectral (MIE) é um classificador espectral que fornece estimativas segundo níveis de significância das áreas mais prováveis do material procurado e foi inicialmente formulado para dados de espectrometria imageada (Carvalho Júnior et al., 2001). Além de gerar uma imagem relativa ao parâmetro de similaridade 0 algoritmo realiza um teste de hipótese para definir quais seriam os melhores valores de corte. Essa informação pode ser utilizada para avaliar o grau de correlação dos materiais em análise, sendo importante em uma análise exploratória. No entanto, esse método ainda não foi utilizado para imagens multiespectrais como 0 do sensor ASTER (Advanced Spaceborne Thermal Emission and Reflection Radiometer).

0 sensor ASTER é proveniente de um esforço cooperativo entre a NASA - Earth Observing System (EOS), o Japan's Ministry of Economy, Trade and Industry (METI) e o Earth Remote Sensing Data Analysis Center (ERSDAC). Os dados desse sensor vêm sendo utilizados em análises de temperatura, emissividade, reflectância e elevação da superfície do terreno. Este sensor consiste de três subsistemas: (a) visivel e infravermeIho próximo (VNIR - 0,5 $\mu \mathrm{m}-0,9 \mu \mathrm{m}$ ), constituído por 3 bandas espectrais com resolução de 15 metros, (b) infravermelho ondas curtas (SWIR - 1,6 $\mathrm{mm}-2,5 \mu \mathrm{m}$ ), com 9 bandas espectrais de resolução espacial de 30 metros e (c) infravermelho termal (TIR), com 5 bandas espectrais de resolução espacial de 90 metros (Abrams, 2000; Fujisada, 1998; Yamaguchi et al., 1998).

0 presente trabalho possui como objetivo adaptar 0 algoritmo MIE, desenvolvido inicialmente para espectrometria imageada, em imagens do sensor ASTER com propósito de distinguir tipos fitofisionômicos do Cerrado.

\section{ÁREA DE ESTUDO}

A área estudo é o Campo de Instrução Militar de Formosa, que possui aproximadamente 115.014 ha e conta com uma extensa área de Cerrado preservado. Situado no município de Formosa (G0), o Campo faz fronteira ao sul com o estado de Minas Gerais e a oeste com 0 Distrito Federal. No contexto hidrográfico a área é limitada pelo rio Preto, a oeste e pelo rio Bezerra, a leste. No presente trabalho a imagem utilizada possui uma dimensão de
$15 \mathrm{~km}$ por $15 \mathrm{~km}$ referente à parte norte do Campo de Formosa (G0) (Figura 1).

A área de estudo está inserida no Grupo Bambuí (Dardenne, 1978), sendo representada por metassiltitos, metassiltitos argilosos, metargilitos e raras intercalações de arcósios no topo. Esse grupo ocorre na porção oriental do Distrito Federal, aflorando em drenagens e em raros cortes de estradas. 0 solo de interflúvio, em quase todo o Distrito Federal e nas proximidades, é o latossolo vermelho-amarelo, mais ou menos ácido; com textura variando de mais de $90 \%$ de areia a mais de $90 \%$ de argila, com pequena porcentagem de silte (Eiten, 2001).

A área localiza-se no domínio dos cerrados que apresenta formações savânicas, campestres e florestais. Essas formas apresentam uma gama de características fisionômicas e estruturas gradativas, com um gradiente de biomassa intimamente relacionado às características de solo e água. No cerrado a forma de menor biomassa é o Campo Limpo e, num gradiente crescente, estão o Campo Sujo, o Campo Cerrado, o Cerrado sentido restrito e 0 Cerradão (Coutinho, 1990). Na área estão presentes a mata galeria (formação florestal), o cerrado sentido restrito (formação savânica) e o campo limpo e campo sujo (formação campestre).

0 Cerrado é caracterizado pela presença de invernos secos e verões chuvosos, um clima Aw segundo a classificação de Köppen. A média anual da precipitação é da ordem de $1500 \mathrm{~mm}$, variando de 750 a $2000 \mathrm{~mm}$, praticamente concentrada na estação chuvosa (outubro a março) (Adámoli et al., 1987).

\section{COMPORTAMENTO ESPECTRAL DA VEGETAÇÃO Comportamento Espectral da Folha}

As folhas são os elementos da vegetação que mais contribuem para 0 sinal detectado pelos sensores ópticos (Colwell, 1974). A energia radiante interage com a estrutura foliar por absorção, espalhamento e transmissão. Uma folha típica é formada por três tecidos básicos: epiderme, mesofilo fotossintético e tecido vascular. 0 mesofilo é formado por células alongadas e de organização compacta (parênquima paliçádico), na parte superior e, na parte inferior, por células de formato irregular e sem arranjo definido (mesofilo esponjoso) além de espaços intercelulares onde ocorrem as trocas gasosas. As células do mesofilo são ricas em cloroplastos, onde se encontram os pigmentos. Os pigmentos das células do parênquima palissádico têm um impacto significativo na absorção e reflectância da luz no visível enquanto que as células do parênquima esponjoso têm um impacto significativo na absorção e reflectância da energia incidente do infravermelho (Jensen, 2000). 


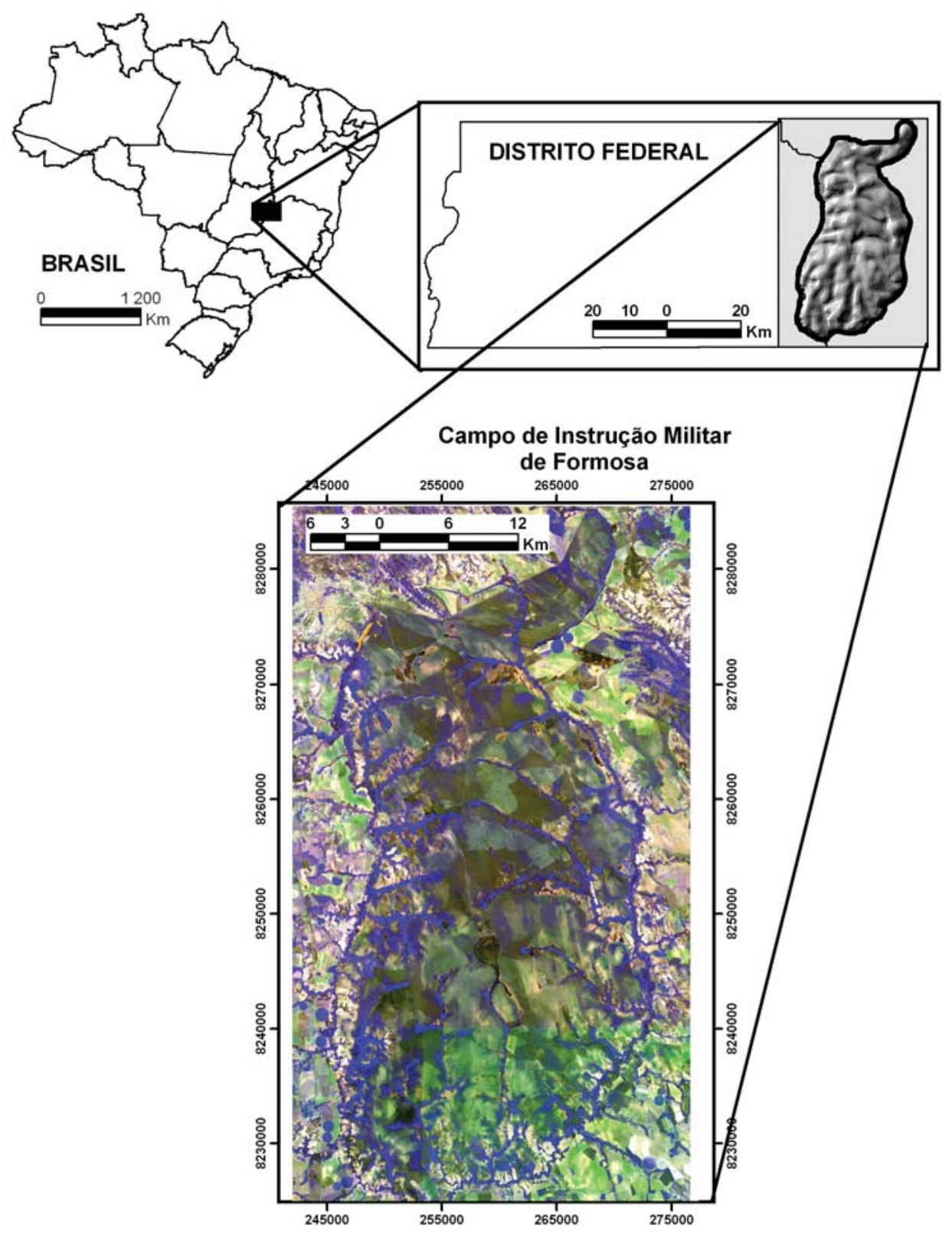

Figura 1 - Localização da área de estudo.

Os pigmentos fotossintéticos (clorofilas e carotenóides) dominam as propriedades espectrais da folha na região do visível (VIS; 0,4 a $0,7 \mu \mathrm{m}$ ), onde a luz é requerida para a fotossíntese (Gates et al., 1965; Gausman, 1974). Nessa faixa do espectro a energia é seletivamente absorvida pela clorofila e convertida em calor ou fluorescência, sendo fotoquimicamente estocada na forma de componentes orgânicos. As folhas verdes sadias são muito eficientes na absorção de energia nos comprimentos de onda do azul (0,4 - 0,5 $\mu \mathrm{m})$ e vermelho (0.6-0.7 $\mu \mathrm{m})$, enquanto os carotenóides absorvem apenas na região do azul.

No infravermelho próximo (NIR; 0,7 a 1,3 $\mu \mathrm{m}$ ) a reflectância da folha verde sadia aumenta significativamente devido ao espalhamento induzido pelas descontinuidades nos índices de refração no mesofilo esponjoso ( $n \sim 1.4$ para a parede celular hidratada, $n \sim 1.3$ para a água e $n=1$ para 0 ar) (Wooley, 1971; Gausman, 1974). A transição abrupta entre a forte absorção nos com- 
primentos de ondas do visível e o espalhamento no infravermelho próximo é conhecido como "red edge" (Curran et al., 1991; Filella \& Peñuelas, 1994; Horler et al., 1983; Lichtenthaler, 1996). As foIhas verdes sadias não utilizam toda a energia do NIR, refletindo (40-60\%) e transmitindo (45-50\%), como forma de controlar 0 aquecimento da folha (Jensen, 2000). No patamar do infravermeIho próximo $(0,97$ a 1,2 $\mu \mathrm{m})$ são descritas duas fracas feições de absorção atribuídas á absorção da água (Curran, 1989; Gao \& Goetz, 1995; Kokaly et al., 1998).

No infravermelho de ondas curtas (SWIR; 1,3 a 2,5 $\mu \mathrm{m}$ ) 0 espectro da folha verde sadia é dominado pelas feições de absorção da água. As principais bandas de absorção ocorrem em 1,40 $\mu \mathrm{m}, 1,94 \mu \mathrm{m}$ e 2,7 $\mu \mathrm{m}$ e as feições secundárias em 0,96 $\mu \mathrm{m}, 1,12 \mu \mathrm{m}, 1,54 \mu \mathrm{m}, 1,67 \mu \mathrm{m}, \mathrm{e} 2,20 \mu \mathrm{m}$ (Knipling, 1970, Wooley, 1971, 1973). Estas feições são oriundas das vibrações de estiramento e de deformação das ligações $0-\mathrm{H}$ da água e de outros elementos químicos (Danks et al., 1984; Curran, 1985, 1989). As feições de absorção provenientes de proteínas, ligninas e celulose aparecem apenas no espectro de folhas secas, devido à redução das feições de absorção da água que mascaram essas feições em folhas verdes (Jacquemoud \& Baret, 1990; Jacquemoud et al., 1996; Kokaly et al., 1998). Desta forma, a assinatura espectral das folhas verdes e sadias na região do visível é dominada pelos pigmentos (clorofilas e carotenóides) enquanto que, no infravermelho ondas curtas é dominada pela água.

Quando a folha entra em processo de senescência ou stress, apresentando redução no teor de clorofilas, a intensidade das bandas de absorção em 0,45 $\mu \mathrm{m}-0,65 \mu \mathrm{m}$ reduz com um aumento simultâneo da reflectância. Essas modificações não são devidas apenas redução das feições de absorção da água, mas também às alterações estruturais que ocorrem na folha quando esta perde umidade (Figura 2).

\section{COMPORTAMENTO ESPECTRAL DO DOSSEL}

Em relação ao dossel, os parâmetros responsáveis pelo comportamento espectral incluem a natureza geométrica (ângulos de iluminação e visada), espectral (outros elementos da vegetação e solo) além de atributos biofísicos dos dosséis (índice de área foliar, IAF e distribuição angular foliar, DAF). Apesar dos padrões das curvas de reflectância de folhas e de dosséis serem bastante similares, diferindo apenas nos valores das intensidades, outros fatores influenciam as medidas de reflectância, dificultando uma correspondência direta de análise entre as curvas espectrais e a imagem formada nos sensores imageadores (Ponzoni, 2001).

No ambiente de Cerrado, uma fonte de variação do dos- sel corresponde à distinção entre 0 estrato herbáceo e a cobertura arbóreo-arbustiva. A vegetação do Cerrado no sentido fisionômico possui as seguintes formações (Ribeiro, 1998): (a) florestais, com predominância de espécies arbóreas, (b) savânicas, áreas com árvores e arbustos espalhados sobre um estrato graminoso, sem a formação de dossel contínuo; e (c) campestre, com predominância de espécies herbáceas e algumas arbustivas, faltando árvores na paisagem. Desta forma, as fitofisononimas são distinguidas inicialmente pela disposição, organização e arranjo dos indivíduos na comunidade, tanto na altura (estrutura vertical) quanto em densidade (estrutura horizontal), seguido das formas de crescimento e as mudanças estacionais.

Essas características são detectadas pelo comportamento espectral do dossel que considera tanto os aspectos biofísicos desse arranjo como também as características espectrais provenientes do comportamento sazonal distinto dos estratos arbóreoarbustivo e herbáceo. Enquanto as plantas herbáceas têm seus eventos fenológicos ligados à estação chuvosa, as plantas lenhosas parecem ser mais independentes da sazonalidade, sendo que muitas delas florescem em plena estação seca apresentando diferenças marcantes na floração e na frutificação (Sarmiento \& Monasterio, 1983; Mantovani \& Martins, 1988).

Em ambientes de savana, a vegetação não fotossinteticamente ativa (VNFA), que inclui principalmente folhas secas e cascas de árvores, apresenta um maior impacto sobre o dossel herbáceo do que sobre o dossel arbóreo (Asner, 1998). Uma pequena porcentagem de VNFA no dossel herbáceo ocasiona grandes alterações na reflectância do dossel. No entanto, poucos estudos focam na importância da VNFA na reflectância do dossel (Asner, 1998; Huemmrich \& Goward, 1997; van Leeuwen \& Huete, 1996), apesar de que em muitos ecossistemas este compreende um componente substancial, como no bioma Cerrado. Conforme Asner (2004) o aumento relativo da VNFA no dossel herbáceo ocasiona: (a) aumento da reflectância com uma maior mudança no NIR (16\%-26\%) e SWIR (24\%$26 \%$ ), (b) redução da feição de absorção ocasionados pelos pigmentos e do platô do NIR presentes nos dosséis verdes, (c) 0 "red edge" torna-se aproximadamente linear sofrendo um achatamento, e (d) emergem feições em 2,075 $\mu \mathrm{m}$ e 2,200 $\mu \mathrm{m}$ provenientes das propriedades ópticas da VNFA (Figura 2).

Imagens multiespectrais como a ASTER possuem bandas espectrais largas e descontínuas, apresentando limitações para análise mais acurada das feições de absorção da vegetação. Em imagens multiespectrais muitos esforços vêm sendo desenvolvidos no intuito de compreender e distinguir os tipos vegetais, prin- 


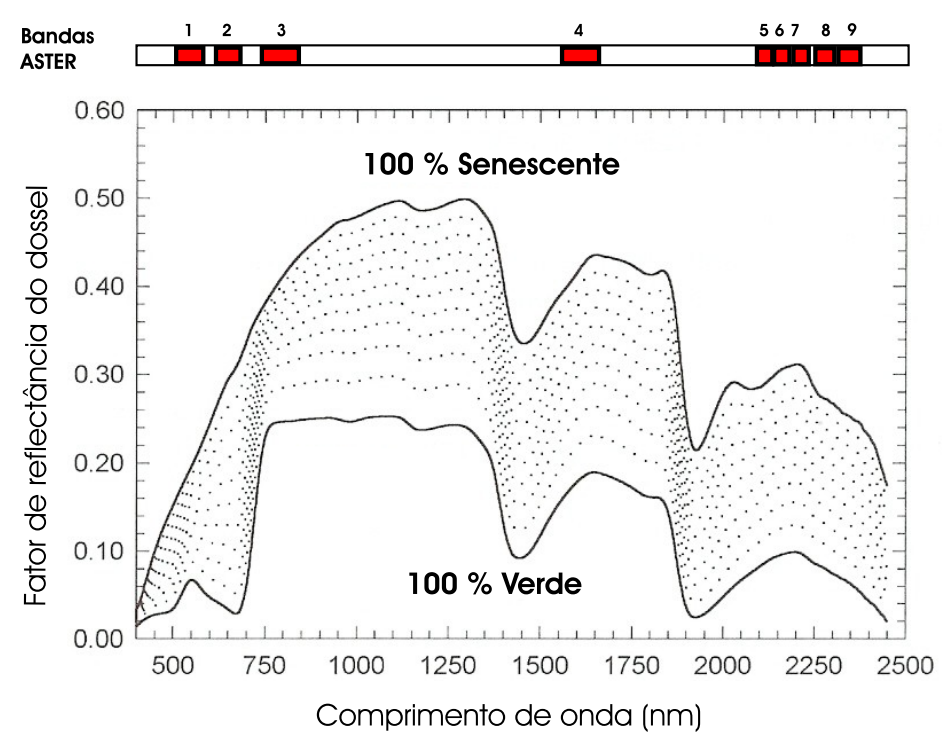

Figura 2 - Demonstração do efeito do aumento do índice de área de vegetação não fotossintética na reflectância do dossel graminoso (Fonte: Asner, 2004).

cipalmente por meio do emprego de índices de vegetação (Asrar et al., 1992; Carlson \& Ripley, 1997, Chen \& Cihlar, 1996; Galvão et al., 1999, Gamon et al., 1995; Van Leeuwen \& Huete, 1996; Huemmrich \& Goward, 1997, Huete, 1988). 0 mais amplamente utilizado é 0 índice de diferença normalizada (NDVI - normalized difference vegetation index) (Rouse et al., 1974). No entanto, o NDVI apresenta limitações em ambientes áridos devido à influência e variação do albedo do solo. Devido a esta limitação foram propostos outros índices com o objetivo de minimizar a influência do solo: índice ajustado ao solo (SAVI - soil - adjusted vegetation index) (Huete, 1988; Huete et al., 1992); 0 índice ajustado ao solo transformado (TSAVI - transformed soil adjusted vegetation index) (Baret et al., 1989), o índice ajustado ao solo resistente à atmosfera (SARVI - soil adjusted atmospherically resistant vegetation index) (Huete \& Liu, 1994); 0 índice ajustado ao solo modificado (MSAVI - modified soil adjusted vegetation index) (Qi et al., 1994); e o índice ajustado ao solo otimizado (OSAVI - optimized soil adjusted vegetation index) (Roundeaux et al., 1996). Além do solo, Van Leewen \& Huete (1996) demonstraram que a presença de VNFA ocasiona uma alta variabilidade nos valores do NDVI e salientam que dificilmente a VNFA apresentam para este índice valores próximos de zero. Estudos utilizando outras faixas dos espectros mostram-se também importantes na distinção dos dosséis vegetais, como por exemplo, o emprego da faixa SWIR na distinção de pastos de idades diferente (Asner et al., 1999; Moran et al., 1994). Portanto, uma alternativa é o emprego de métodos que considerem de forma conjunta a importância relativa de cada banda na caracterização dos tipos vegetais, diferentemente dos índices que ficam restritos a uma determinada feição. Os classificadores espectrais permitem essa abordagem uma vez que 0 processamento é realizado utilizando todo o espectro.

\section{MÉTODO DE INDENTIFICAÇÃO ESPECTRAL}

Os classificadores espectrais baseiam-se na comparação do espectro da imagem (EI) com um espectro de referência (ER), proveniente de bibliotecas espectrais ou de membros finais das imagens. A comparação é realizada por intermédio de um critério de similaridade. Desta forma, a identificação do material procurado é feita segundo o ajuste obtido entre as duas curvas espectrais. Quanto melhor for o ajuste, maior será a probabilidade da existência do material de referência no pixel da imagem (Carvalho Júnior et al., 2003).

0 objetivo desse tipo de classificação é responder aonde existe 0 espectro procurado, sem importar com a sua quantidade no pixel. Basicamente procura-se identificar a existência ou não do espectro de referência. Esse fato torna-se importante, pois em muitos casos ocorrem equívocos com a utilização de métodos de quantificação com o objetivo de identificar ou vice-versa. Os procedimentos de quantificação apresentam resultados coerentes quando o material procurado apresenta um predomínio no pixel, em caso contrário, quando as proporções do material de interesse são baixas esses métodos assinalam a inexistência do material decorrendo em erro.

Dentre os métodos de classificação espectral destacam-se: 
(a) método Spectral Angle Mapper (SAM) que utiliza 0 arcocosseno do coeficiente de correlação não normalizado (Kruse et al., 1993); (b) o Spectral Correlation Mapper (SCM) que utiliza o coeficiente de correlação normalizado pela média (CarvaIho Júnior \& Meneses, 2000); (c) o TRICORDER que consiste em um sistema especialista onde utiliza várias técnicas para a identificação do elemento procurado sendo um integrante importante 0 coeficiente de determinação (Clark \& Swayze, 1995) e a remoção do contínuo (Clark \& Roush, 1984); e (d) 0 método Spectral Feature Fitting (SFF) que utiliza 0 erro o padrão da regressão linear conjuntamente com a remoção do contínuo (ENVI, 1997).

No entanto, existe a dificuldade de determinar o valor do grau de similaridade que permita validar estatisticamente a existência do elemento procurado de forma automatizada. Esse limite de detecção é variável e dependente do material analisado e da correlação existente entre os materiais em análise.

0 Método de Identificação Espectral (MIE) estabelece critérios para definir os valores de cortes. Este método fornece um novo índice de similaridade e três estimativas segundo níveis de significância das possíveis áreas do material procurado (Carvalho Júnior et al., 2001). Esse método fundamenta-se em dois procedimentos estatísticos: ANOVA (Davis, 1973; Steel \& Torrie, 1980; Vieira, 1988; Souza, 1998) e coeficiente SCM (Carvalho Júnior \& Meneses, 2000).

Pela análise de variância, a consideração de uma curva observada como pertencente ao grupo de referência é realizada através de um teste de hipóteses. Neste teste são avaliadas duas alternativas:

$$
\begin{aligned}
& \mathrm{H} 0: \beta=0 \\
& \mathrm{H} 1: \beta \neq 0
\end{aligned}
$$

Neste caso, se a hipótese Ho confirmar o espectro da imagem não é similar ao espectro padrão. Não é possível estabelecer uma regra que permita rejeitar $\mathrm{Ho}$, sem que se esteja sujeito a um dos dois tipos de erro: (a) tipo 1 - consiste em rejeitar $\mathrm{Ho}$, sendo que Ho é verdadeira; e (b) tipo 2 - consiste em aceitar $\mathrm{Ho}$, sendo que Ho é falso. A probabilidade da ocorrência do erro tipo I é denominada, no teste de hipótese, como nível de significância $(\alpha)$. Um nível de significância de 10\% consiste em dizer que existe uma probabilidade de $10 \%$ dos dados possuírem $\beta=0$ e serem considerados como $\beta \neq 0$. Isto significa que $10 \%$ dos dados não são oriundos da população do padrão e que erroneamente estão classificadas como dela pertencente. Para a validação ou não da hipótese é empregado o teste F onde a média da soma dos quadrados da regressão (MQr) é dividido pela a média da soma dos quadrados do desvio (MQd).

$$
\mathrm{F}=\mathrm{MQr} / \mathrm{MQd}
$$

0 procedimento do teste consiste em rejeitar Ho para todo valor de $\mathrm{F}$ calculado igual ou maior do que 0 valor estabelecido segundo uma tabela de distribuição (Vieira, 1988). 0 valor de $F$ segundo a tabela de distribuição é função do nível de significância $(2,5 \%, 5 \%$ e $10 \%)$ e dos graus de liberdade do numerador $e$ denominador. A Tabela 1, conhecida como tabela de análise de variância, consiste em um resumo dos procedimentos estatísticos para a realização dessa análise. 0 resultado de $F$ obtido para cada pixel é comparado com os valores da tabela de distribuição F (Davis, 1973; Vieira, 1988). Se o valor for menor não se trata do alvo pretendido.

0 grau de liberdade do denominador é expresso como sendo (N-2). A utilização literal do número de bandas para o cálculo dos graus de liberdade gera-se resultados inconsistentes. Observa-se que para o processamento das imagens 0 emprego dos graus de liberdade do desvio deve ser bem inferior do que o recomendado devendo ser entre um ou dois. Essa característica também foi verificada para imagens hiperespectrais e se deve, provavelmente, devido à alta redundância de informações.

Como a análise de variância de uma regressão linear não é sensível à presença da correlação negativa é utilizado como complemento o classificador espectral Spectral Correlation Mapper (Carvalho Júnior \& Meneses, 2000). Desta forma, pode-se segmentar as áreas com correlação negativa e adicioná-las aos dados obtidos com a ANOVA restringindo as áreas de real interesse. 0 presente método gera imagens binárias, relativas à existência ou não da informação pretendida, dentro de uma margem de significância de 2,5\%, 5\% e 10\%.

0 Método de Identificação Espectral (MIE) foi implementado, em linguagem IDL, dentro do programa ENVI. Esse programa utiliza um espectro de referência e a imagem a ser analisada. 0 programa gera quatro imagens de saída, sendo que as primeiras três são referentes aos níveis de significância de 2,5\%, 5\% e 10\% do método e a última, à imagem do índice de similaridade. A imagem dos níveis de significância é de caráter binário podendo ser utilizada como uma máscara para delimitar as áreas de interesse.

\section{PROCESSAMENTO DIGITAL DAS IMAGENS ASTER}

A metodologia aplicada para o processamento digital das imagens ASTER constou das seguintes etapas: (a) pré-processamento, (b) identificação dos membros finais e (c) aplicação do método MIE. A imagem utilizada no presente trabalho é do dia 21-10-2000 referente ao final da época seca neste ano. 
Tabela 1 - ANOVA para uma Regressão Linear Simples (Davis, 1973).

\begin{tabular}{|c|c|c|c|c|}
\hline Origem da Variação & Soma dos quadrados & Graus de liberdade & Média dos Quadrados & F Teste \\
\hline Regressão Linear & SQr & 1 & MQr & \multirow{2}{*}{ MQr/MQd } \\
\hline Desvio & SQd & $\mathrm{N}-2$ & MQd & \\
\hline Total da Variação & SQt & $\mathrm{N}-1$ & & \\
\hline
\end{tabular}

As nove imagens utilizadas (VNIR e SWIR) foram adquiridas já corrigidas do efeito atmosférico, correspondendo aos produtos de alto nível do sensor ASTER referente à especificação AST07 (JPL, 2001; Thome et al., 1998). A correção atmosférica remove os efeitos devido às mudanças de geometria satélite - sol e das condições atmostéricas.

Devido a diferenças de resolução espacial entre as imagens do VNIR (15 m) e as do SWIR (30m) foi necessário a realização prévia de uma compatibilização entre as imagens. Essa normalização foi obtida pela duplicação dos pixels das imagens do SWIR. Desta forma, os pixels relativos às imagens SWIR adquirem uma dimensão de 15m compatível à resolução espacial das imagens VNIR. A nona banda do sensor ASTER não foi considerada no trabalho devido a problemas de calibração. Desta forma, as oito bandas restantes foram integradas em uma única imagem (Figura 3). Essa junção permite reconstituir o espectro de um pixel, etapa imprescindível para 0 emprego dos classificadores espectrais. Nesta concepção, a identificação de um elemento parte da reconstrução dos membros finais que podem estar contidos dentro de um mesmo pixel em diferentes proporções, 0 que difere das classificações que utilizam algoritmos de análise de grupos onde se estabelecem categorias taxonômicas em que cada classe recebe um único rótulo. Essa técnica incorpora informações espectrais a partir de um número ilimitado de bandas simultaneamente, considerando todas as características da curva espectral, 0 que 0 torna sensível a diferentes concentrações.

A identificação dos membros finais consiste em uma etapa exploratória, ou seja, de uma primeira avaliação dos materiais existentes na área. Além disso, esse procedimento permite identificar e avaliar os efeitos relativos aos fatores físicos ambientais presentes na cena. A identificação dos membros finais foi realizada conforme a metodologia proposta por Boardman \& Kruse (1994) constituída por três etapas: (a) redução da dimensão espectral utilizando a técnica de Minimization Noise Fraction (MNF) (Green et al., 1988), (b) redução da dimensão espacial utilizando o método Pixel Purity Index (PPI) (Boardman \& Kruse, 1994; Boardman et al., 1995) e (c) identificação utilizando um visualizador n-dimensional.
A partir da identificação dos membros finais foi aplicado 0 método MIE sobre a imagem. Com o propósito de aplicar 0 método MIE em imagens multiespectrais do sensor ASTER foi necessário definir os valores de graus de liberdade do denominador $(\mathrm{N}-2)$ que melhor se ajustavam aos dados ficando no caso entre os valores $1 \mathrm{e} 2$.

\section{RESULTADOS}

\section{Análise dos Membros Finais}

Os membros finais identificados correspondem a uma determinada fitofisionomia presente na área. A mata galeria (formação florestal) constituída por espécies arbóreas compõe um dossel contínuo perenofólia que se caracteriza por um comportamento espectral de VFA.

0 cerrado sentido restrito no período da seca descreve um comportamento espectral do dossel que retrata a mistura da VFA e VNFA uma vez que é formado por árvores e arbustos espalhados com presença de VFA e VNFA sobre um estrato graminoso caracterizado por VNFA.

0 campo sujo e o campo limpo (formação campestre), onde predominam espécies graminosas, apresentam sazonalmente as partes aéreas completamente secas, 0 caracteriza um comportamento espectral típico de VNFA. Nesse estrato observa-se uma maior incidência de queimadas devido ao acúmulo de biomassa combustível (Batmanian, 1983; Coutinho, 1990). Desta forma, nas áreas que sofreram queimadas observa-se um aumento da contribuição do solo exposto no comportamento espectral.

Do ponto de vista do sensoriamento remoto, a quantidade relativa de VNFA e VFA é preponderante na distinção entre as fitofisionomias de mata de galeria, cerrado e campo. A Figura 4 apresenta os espectros dos membros finais encontrados na área.

\section{Análise da Imagem do Fator de Similaridade MIE}

0 fator de similaridade MIE apresenta como valor máximo às áreas com nível de significância igual a 2,5\%, enquanto que os valores mais baixos são relativos as áreas com SCM abaixo de $40 \%$ e de fator $F$ abaixo de 5 . As imagens de similaridades 
VNIR (15x15)
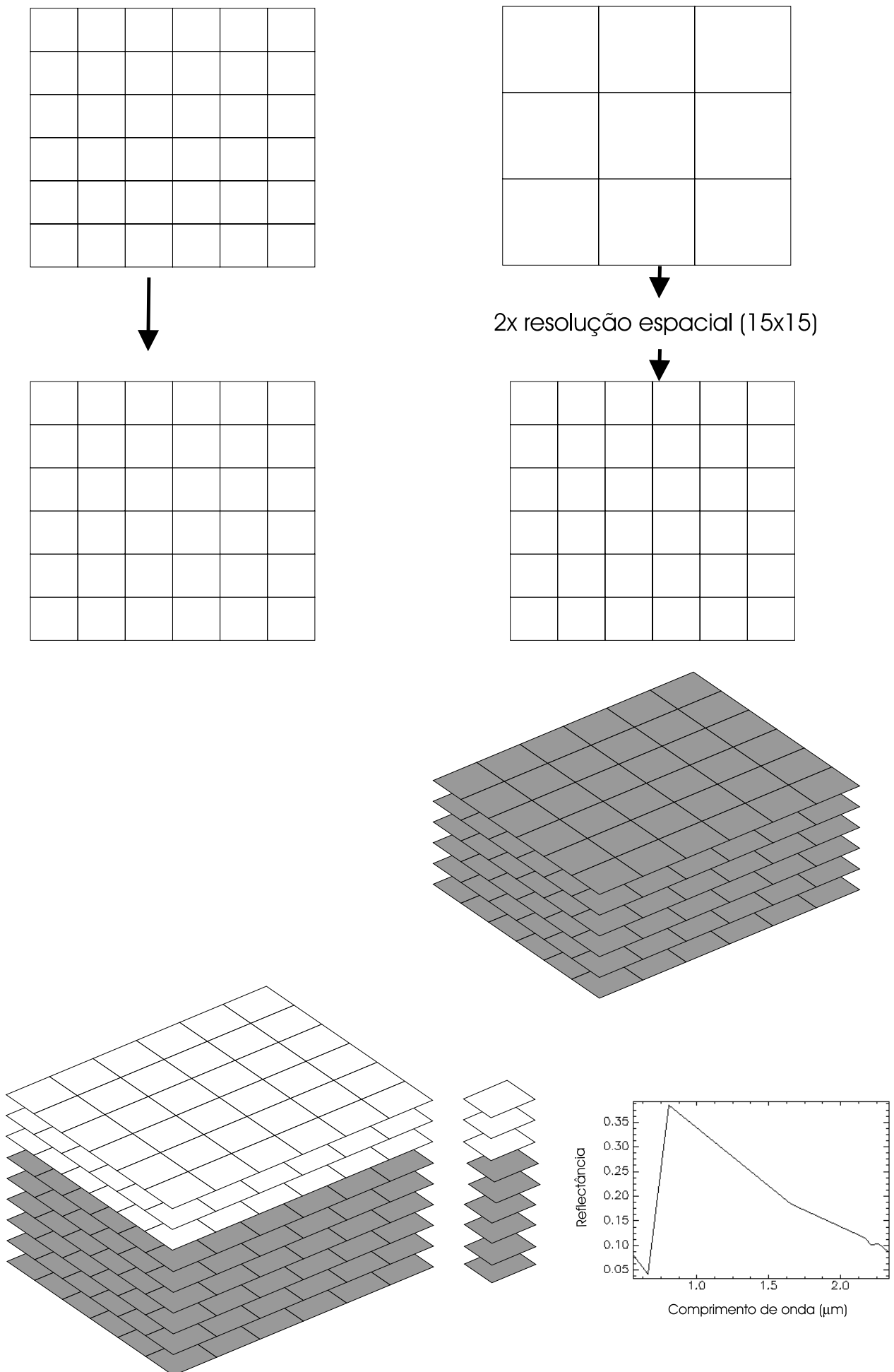

Figura 3 - Compatibilização das resoluções espaciais entre as imagens VNIR e SWIR. 
IMAGEM DO FATOR DE SIMILARIDADE

CURVA ESPECTRAL
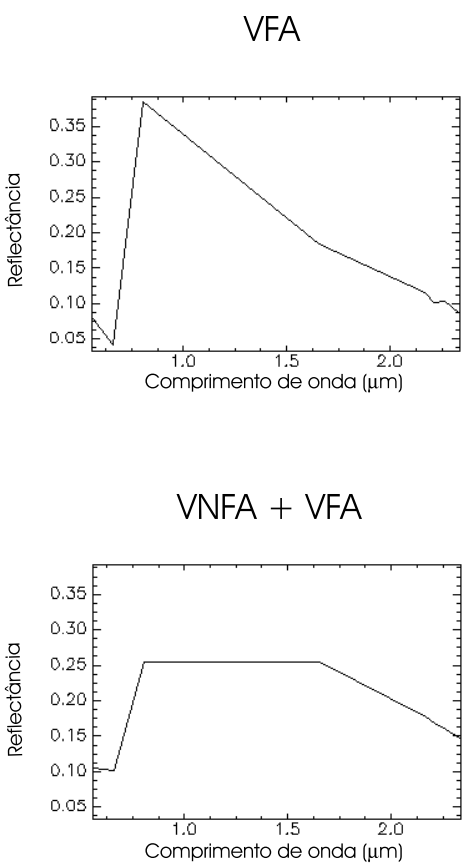

VNFA

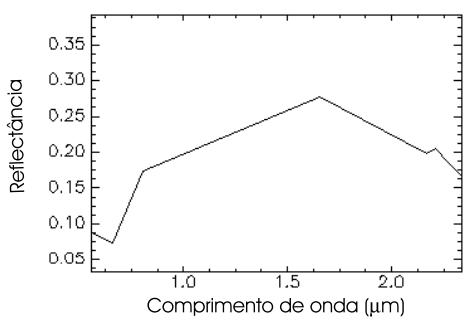

SOLO +VNFA

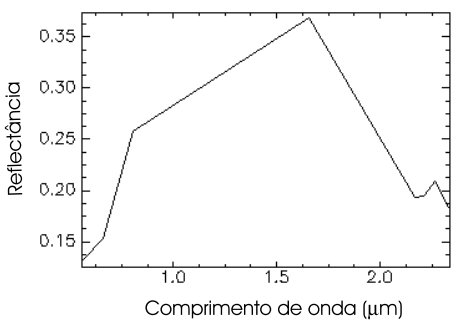

Modelo 1
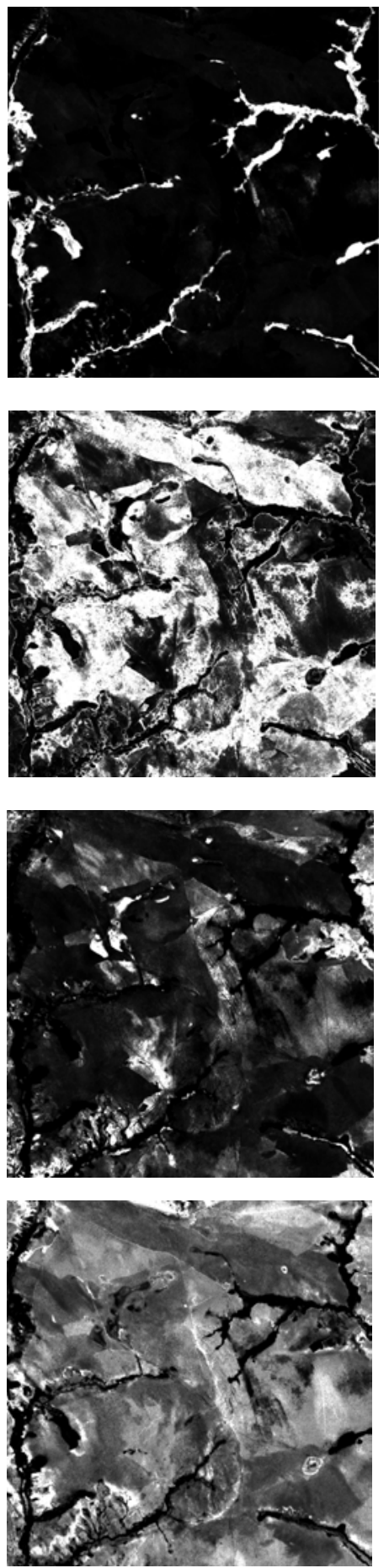

Modelo 2
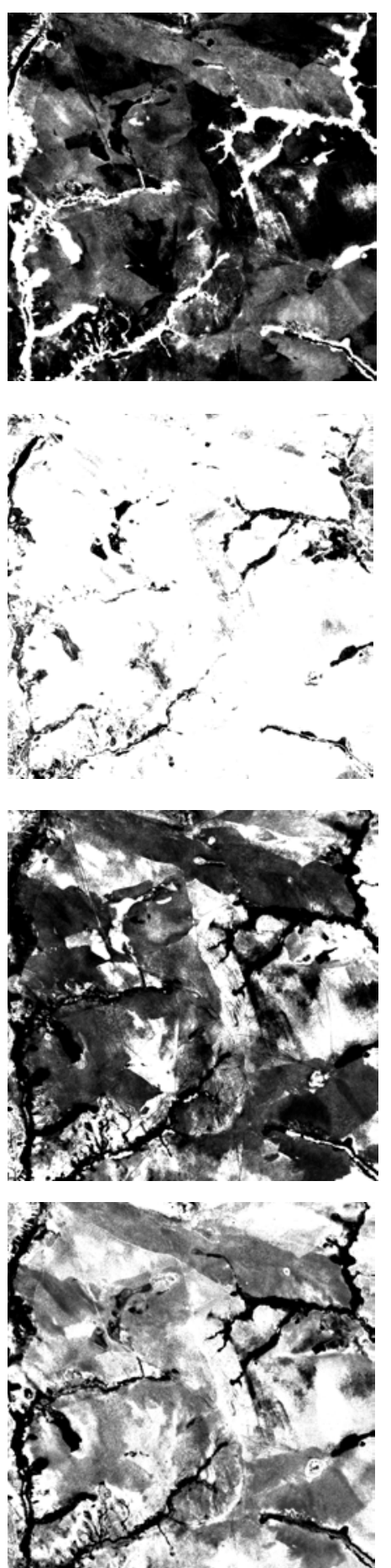

Figura 4 - Espectros dos membros finais e suas respectivas imagens do parâmetro de similaridade considerando duas situações representadas por tipo 1 e 2. 

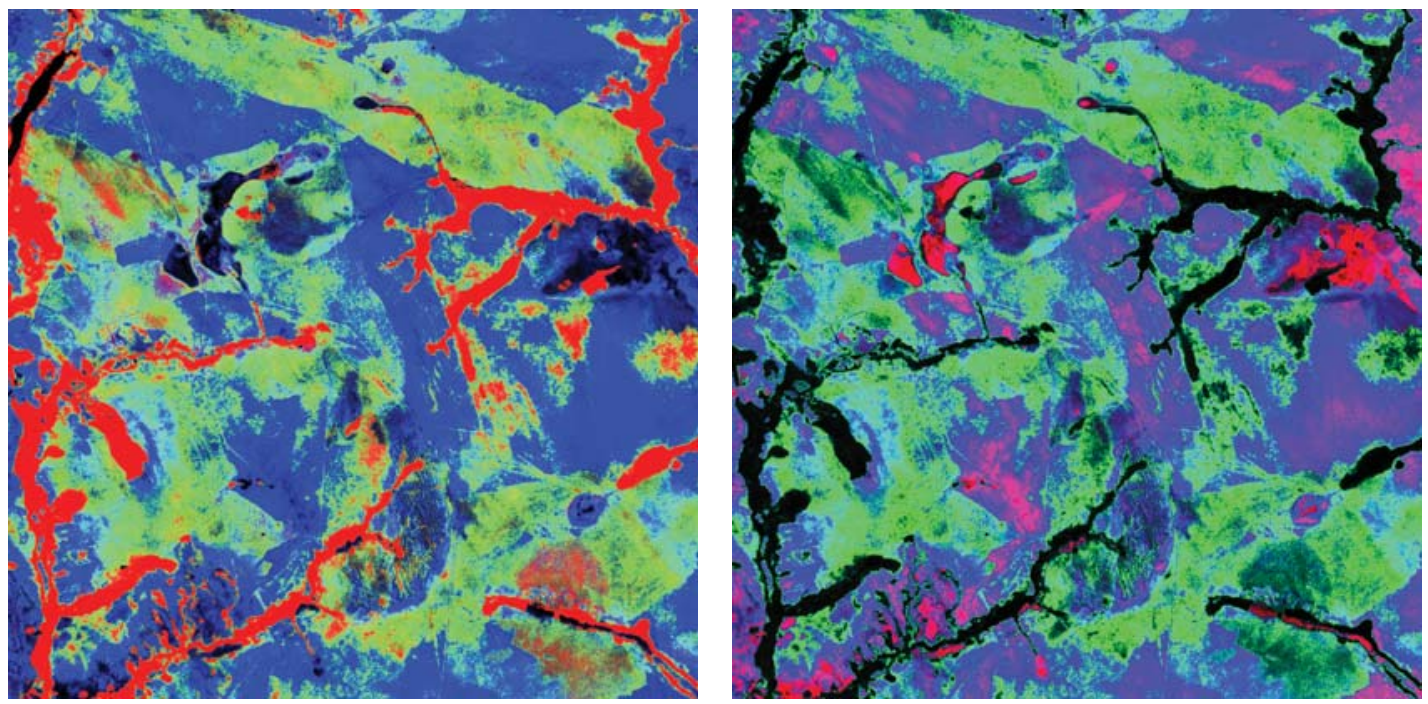

Figura 5 - Composição colorida das imagens do parâmetro de similaridade: (a) VFA (R)/NFA+VNFA (G)/ VNFA (B); (b) VNFA+Solo (R)/ VFA+VNFA (G), VNFA+Solo (B).

são geradas considerando os graus de liberdade do denominador igual a um (modelo 1) como também dois (modelo 2) (Figura 5). Observa-se que 0 modelo 1 restringe mais 0 alvo procurado. 0 modelo 2 melhor realçou a VFA (mata galeria) e VNFA (campos) enquanto que o modelo 1 as misturas com a VNFA (cerrado sentido restrito).

Existe pouca sobreposição entre os alvos com exceção da VNFA e a VNFA + Solo que apresentam muita similaridade espectral e correspondem às formações campestres. Para distingui-los é necessário restringir a faixa espectral às bandas do SWIR 5-67-8 que apresentam diferenciações para as feições de absorção.

0 emprego de composiçã̃o colorida com essas imagens permite uma excelente discriminação visual dos diferentes alvos que compõe a área. A presença de variações abruptas e geométricas na vegetação corresponde aos limites de antigas fazendas que foram desapropriadas para a construção do Campo de Instrução e efeitos de queimadas.

\section{Análise das Imagens dos Graus de Significância}

0 presente método gera imagens binárias, relativas à existência ou não da informação pretendida, dentro de uma margem de significância de 2,5\%, 5\% e 10\%. Como descrito, anteriormente, foram adotados como graus de liberdade para a média da soma dos quadrados do desvio os valores 1 (modelo 1) e 2 (modelo 2).

Na Figura 6 são apresentados as imagens referentes ao modelo 1 enquanto que a Figura 7 exibe as imagens do modelo 2. Observa-se no modelo 1 que 0 grau de significância $10 \%$ permite uma boa demarcação das áreas de mata galeria enquanto que 0 grau de significância 2,5\% restringe muito a área de ocorrência. Em contraposição, no modelo 2 ocorre uma pronunciada expansão da área da VFA, atingindo no grau de significância $10 \%$ toda a área relativa a mistura entre VFA + VNFA. Estes dois cenários são importantes, pois evidenciam localidades onde existe a predominância de espectros praticamente puros e outros onde ocorrem misturas espectrais com VNFA.

As imagens relativas aos graus de significância para a VNFA são apresentados nas Figuras 8 e 9 . De forma similar ao apresentado para a VFA, 0 modelo 1 apresenta para VNFA uma boa demarcação no grau de significância 10\%. As imagens relativas aos graus de significância menores restringem aos pixels com características mais puras em relação ao espectro padrão. № modelo 2 observa-se a ampliação para as localidades com presença de misturas espectrais da VFNA. No grau de significância 15\% as áreas desconsideradas são apenas as localidades com mata galeria.

\section{CONCLUSÃO}

0 método MIE para obter resultados consistentes com as informações de campo teve que adotar para a média da soma dos quadrados do desvio valores de 1 e 2 graus de liberdade. As imagens do parâmetro de similaridade do MIE permitem um alto contraste visual destacando os alvos procurados. As principais diferenças espectrais entre a mata de galeria, cerrado sentido restrito e campo na imagem correspondem ao contraste entre VFA 

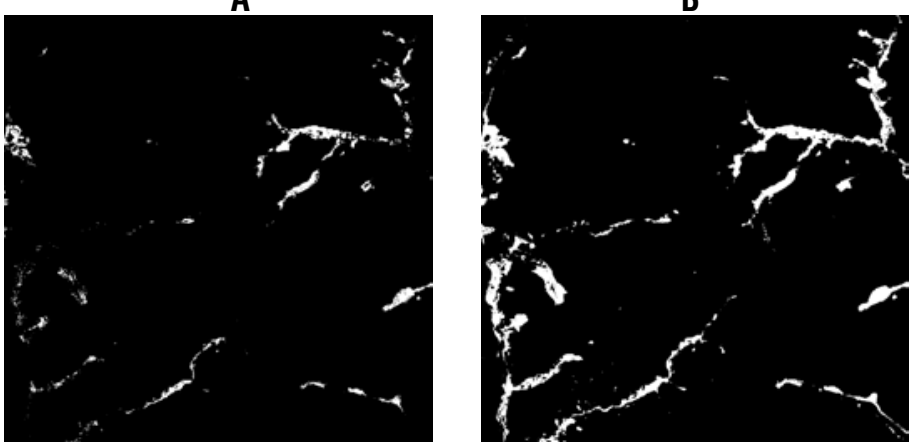

Figura 6 - Imagens dos graus de significância para VFA considerando o modelo 1: (a) 2,5\%, (b) 5\% e (c) 10\%.
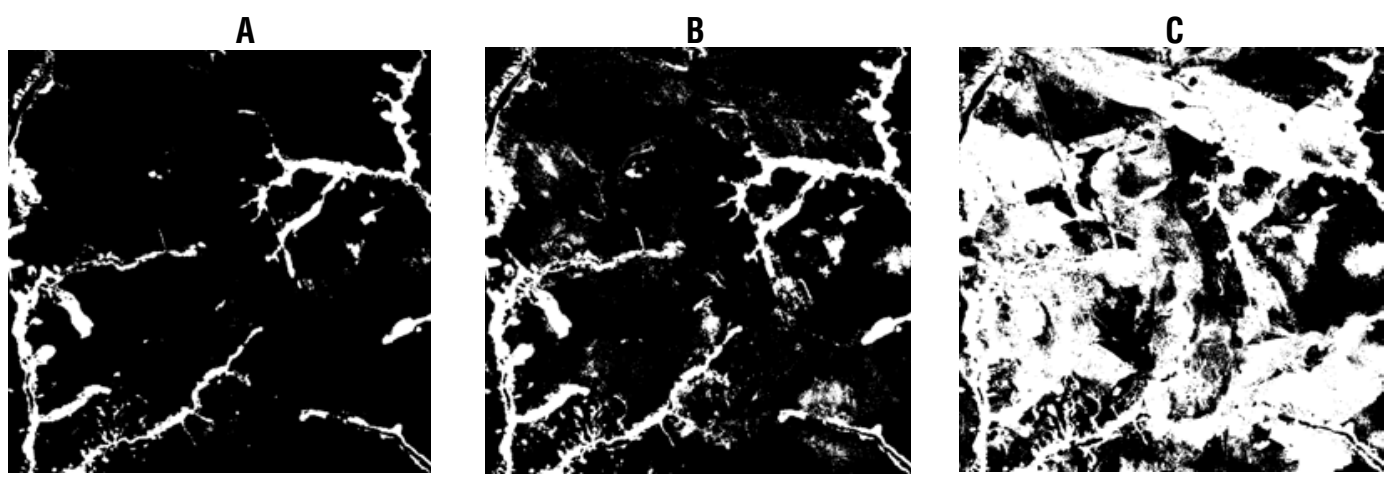

Figura 7 - Imagens dos graus de significância para VFA considerando o modelo 2: (a) 5\%, (b) 10\% e (c) 15\%.
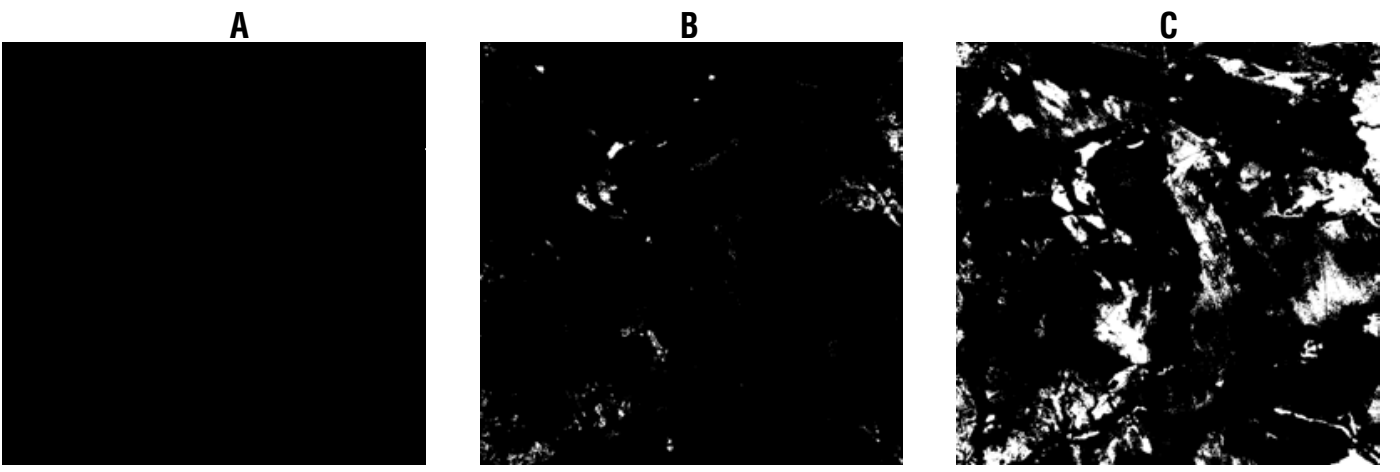

Figura 8 - Imagens dos graus de significância para VNFA considerando o modelo 1: (a) 2,5\%, (b) 5\% e (c) 10\%.
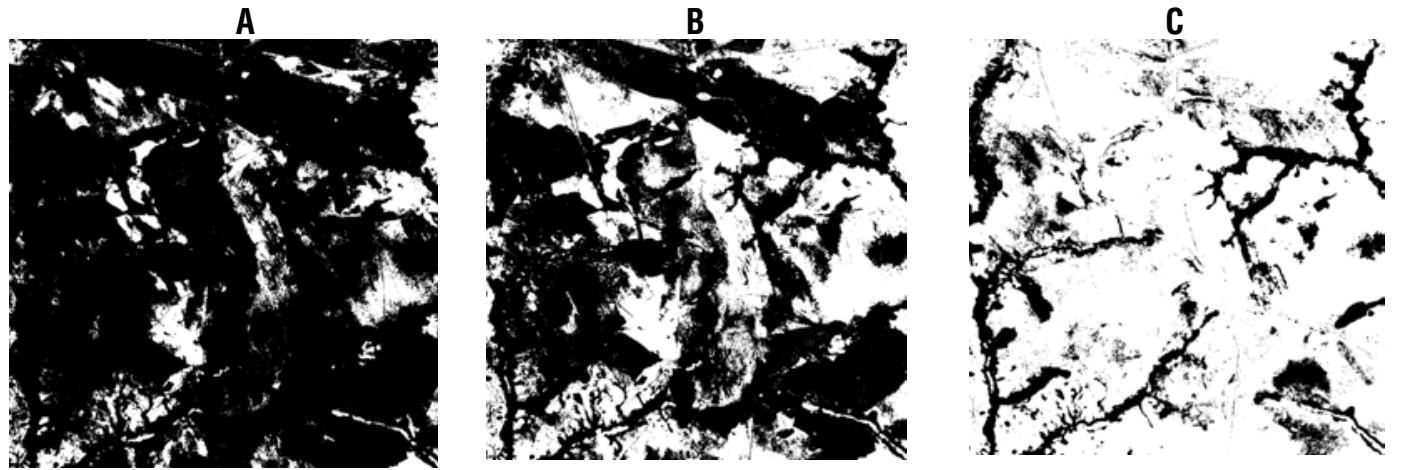

Figura 9 - Imagens dos graus de significância para VNFA considerando o modelo 2: (a) 2,5\%, (b) 5\% e (c) 10\%. 
e VNFA. 0 emprego de composições coloridas permite delimitar as diferentes zonas ambientais.

As imagens relativas aos graus de significância são bastante úteis no procedimento de análise exploratória. Observa-se uma variação das imagens conforme 0 espectro de referência 0 que torna difícil uma padronização na interpretação dessas imagens de saída. No entanto, a partir de uma análise da série de imagens pode-se identificar o limite mais adequado para o espectro procurado.

\section{AGRADECIMENTOS}

Os autores agradecem ao Exército Brasileiro por permitir e auxiliar a realização dos trabalhos dentro do Campo de Instrução Militar de Formosa, ao CNPq pelo financiamento de bolsas de pesquisa e aos consultores da revista de geofísica.

\section{REFERÊNCIAS}

ABRAMS M. 2000. The Advanced Spaceborne Thermal Emission and Reflection Radiometer (ASTER): data products for the high spatial resolution imager on NASA's Terra platform. International Journal of Remote Sensing, 21(5): 847-859.

ADÁMOLI J, MACEDO J, AZEVEDO LG \& NETTO JM. 1987. Caracterização da região dos Cerrados. In: W. J. GOEDERT (ed). SoIos dos Cerrados: tecnologias e estratégias de manejo. Planaltina: EMBRAPA - CPAC; São Paulo: NOBEL, p. 33-98.

ADAMS JB, SABOL DE, KAPOS V, ALMEIDA FILHO R, ROBERTS A, SMITH MO \& GILLESPIE AR. 1995. Classification of multispectral images based on fractions of endmembers: application to land-cover change in the Brazilian Amazon. Remote Sensing of Environment, 52: 137-154.

ASNER GP. 1998. Biophysical and biochemical sources of variability in canopy reflectance. Remote Sensing of Environment, 64: 234-253.

ASNER GP. 2004. Biophysical remote sensing signatures of arid and semiarid ecosystems. In: Ustin S. (ed.). Manual of remote sensing. 3. ed., 4. v. John Wiley \& Sons, Inc. p. 53-109.

ASNER GP, TOWNSEND AR \& BUSTAMANTE MC. 1999. Spectrometry of pasture condition and biogeochemistry in the Central Amazon. Geophysical Research Letter, 26(17): 2769-2772.

ASRAR GF, MYNENI RB \& CHOUDBURY BJ. 1992. Spatial heterogeneity in vegetation canopies and remote sensing of absorbed photosynthetically active radiation: a modeling study. Remote Sensing of Environment, 41: 85-103.

BARET F, GUYOT G \& MAJOR D. 1989. TSAVI: a vegetation index which minimizes soil brightness effects on LAl of APAR estimation. In: Canadian Symposium on Remote Sensing and IGARSS '90, 12., Vancouver, Canada. Proceedings, 10-14 July, 4 p.
BATMANIAN GJ. 1983. Efeitos do fogo sobre a produção primária e a acumulação de nutrientes do estrato rasteiro de um cerrado. Dissertação (Mestrado em Ecologia) - Universidade de Brasília, Brasília, 78 p.

BOARDMAN JW \& KRUSE FA. 1994. Automated spectral analysis: A geologic example using AVIRIS data, north Grapevine Mountains, Nevada. In: ERIM - Thematic Conference on Geologic Remote Sensing, 10, Ann Arbor, Ml. Proceedings, Ann Arbor: Environmental Research Institute of Michigan, 1: 407-418.

BOARDMAN JW, KRUSE FA \& GREEN RO. 1995. Mapping target signatures via partial unmixing of AVIRIS data: In: Annual JPL Airborne Geoscience Workshop, 5., Pasadena, CA. Summaries, JPL Publication. 95(1): 23-26.

CARLSON TN \& RIPLEY DA. 1997. On relation between NDVI, fraction vegetation cover, and leaf area index. Remote Sensing of Environment, 62: 241-252.

CARVALHO JÚNIOR OA, CARVALHO APF, MENESES PR \& GUIMARÃES RF. 2001. Spectral Identification Method (SIM): a new classifier based on the ANOVA and Spectral Correlation Mapper (SCM) Methods. In: JPL AIRBORNE EARTH SCIENCE WORKSHOP, 10, Pasadena, CA. Proceedings, JPL Publication 02, p. 67-72.

CARVALHO JÚNIOR OA, CARVALHO APF, GUIMARÃES RF \& MENESES PR. 2003. Mistura espectral: (II) classificadores espectrais para identificação. Espaço \& Geografia, 6(1): 177-197.

CARVALHO JÚNIOR OA \& MENESES PR. 2000. Spectral Correlation Mapper (SCM): an improving Spectral Angle Mapper. In: Annual JPL Airborne Earth Science Workshop, 9., Pasadena, CA. Proceedings, JPL Publ. 00-18, 65-74.

CHEN JM \& CIHLAR J. 1996. Retrieving Leaf Area Index of Boreal Conifer Forest Using Landsat TM Images. Remote Sensing of Environment, 55: 153-162.

CLARK RN \& ROUSH TL. 1984. Reflectance Spectroscopy: Quantitative analysis techniques for remote sensing applications. Journal of Geophysical Research, 89: 6329-6340.

CLARK RN \& SWAYZE GA. 1995. Mapping minerals, amorphous materials, environmental materials, vegetation, water, ice and snow, and other materials: the USGS Tricorder algorithm. In: JPL Airborne Earth Science Workshop, 5, Pasadena, CA. Summaries, JPL Publ. 95-1, p. 39-40.

COLWELL JE. 1974. Vegetation canopy reflectance. Remote Sensing of Environment, 3: 175-183.

COUTINHO LM. 1990. Fire in the ecology of the Brazilian Cerrado. In: J. G. GOLDAMMER, J. C. (ed.). Fire in the tropical biota: ecosystem processes and global challenges. Berlim: Springer-Verlag, p. 82-105.

CURRAN PJ. 1985. Principles of remote sensing. London: Logman Scientific and Technical, $282 \mathrm{p}$.

CURRAN PJ. 1989. Remote sensing of foliar chemistry. Remote Sensing of Environment, 30: 271-278. 
CURRAN PJ, DUNGAN JL, MACLER BA \& PLUMMER SE. 1991. The effect of a red leaf pigment on the relationship between red edge and chlorophyll concentration. Remote Sensing of Environment, 35(1): 6976.

DANKS SM, EVANS EH \& WHITTAKER PA. 1984. Photosynthetic systems: structure, function and assembly. New York: John Wiley \& Sons Ltd, $174 \mathrm{p}$.

DARDENNE MA. 1978. Síntese sobre a estratigrafia do Grupo Bambuí no Brasil Central. In: Congr. Bras. Geoc., 30. Recife, Anais, SBG. 2: 597-610.

DAVIS JC. 1973. Statistics and data analysis in geology. New York: John Willey \& Sons, Inc. $550 p$

EITEN G. 2001. Vegetação natural do Distrito Federal. Brasília: UnB: SEBRAE. $162 \mathrm{p}$.

ENVIB). 1997. Tutorials better solutions. Consulting Limited Liability Company Lafayette, Colorado, USA, $370 \mathrm{p}$.

FILELLA I \& PEÑUELAS J. 1994. The red edge position and shape as indicators of plant chlorophyll content, biomass and hydric status. International Journal of Remote Sensing, 15(7): 1459-1470.

FUJISADA H, SAKUMA F, ONO A \& KUDOH M. 1998. Design and preflight performance of ASTER instrument protoflight model. IEEE Transactions on Geoscience and Remote Sensing, 36(4): 1152-1160.

GALVÃO LS, VITORELLO I \& ALMEIDA FILHO R. 1999. Effects of bands positioning and bandwitdth on NDVI measurements of tropical savannas. Remote Sensing of Environment, 67: 181-193.

GAMON JA, FIELD CB, GOULDEN M, GRIFFIN K, HARTLEY A, JOEL G, PENUELAS J \& VALENTINI R. 1995. Relationships between NDVI, canopy structure, and photosynthetic activity in three Californian vegetation types. Ecological Applications, 5: 28-41.

GAO BC \& GOETZ AFH. 1995. Retrieval of equivalent water thickness and information related to biochemical components of vegetation canopies from AVIRIS data. Remote Sensing of Environment, 52: 155-162.

GATES DM, KEEGAN HJ, SCHLETER JC \& WEIDNER VR. 1965. Spectral properties of plants. Applied Optics, 4(1): 11-20.

GAUSMAN HW. 1974. Leaf reflectance of near infrared. Photogrammetric Engineering, 40: 183-191.

GREEN AA, BERMAN M, SWITZER P \& CRAIG MDA. 1988. Transformation for ordering multispectral data in terms of images quality with implications for noise removal. IEEE Transactions on Geoscience and Remote Sensing, 26(1): 65-74.

HORLER DNH, DOCKRAY M \& BARBER J. 1983. The red edge of plant leaf reflectance. International Journal of Remote Sensing, 4: 273-288.
HUEMMRICH KF \& GOWARD SN. 1997. Vegetation canopy PAR absorptance and NDVI: an assessment for ten tree species with the SAIL model. Remote Sensing of Environment, 61: 254-269.

HUETE AR. 1988. A soil-adjusted vegetation index (SAVI). Remote Sensing of Environment, 25: 295-309.

HUETE AR, HUA G, QI J, CHEHBOUNI A \& VAN LEEUWEN WJD. 1992. Normalization of multidirectional red and NIR reflectances with the SAVI Remote Sensing of Environment, 41(2-3): 143-154.

HUETE AR \& LIU HQ. 1994. An error and sensitivity analysis of the atmospheric and soil correcting variants of the NDVI for the MODIS-EOS. IEEE Transactions on Geoscience and Remote Sensing, 32: 897-905.

JACQUEMOUD S \& BARET F. 1990. PROSPECT: A model of leaf optical properties spectra. Remote Sensing of Environment, 34: 75-91.

JACQUEMOUD S, USTIN SL, VERDEBOUT J, SCHMUCK G, ANDREOLI G \& HOSGOOD B. 1996. Estimating leaf biochemistry using the PROSPECT leaf optical properties model. Remote Sensing of Environment, 56: 194-202.

JENSEN JR. 2000. Remote sensing of environment: an Earth resource perspective. New Jersey: Prentice Hall Series in Geographical Information, $544 p$.

JET PROPULSION LABORATORY (JPL). 2001. ASTER higher-level product user guide, JPL D-20062, $80 \mathrm{p}$

KNIPLING EB. 1970. Physical and physiological basis for the reflectance of visible and near-infrared radiation from vegetation. Remote Sensing of Environment, 1: 155-159.

KOKALY RF, CLARK RN \& LIVO KE. 1998. Mapping the biology and mineralogy of Yellowstone National Park using imaging spectroscopy. In: JPL Airborne Earth Science Workshop, 7., Pasadena, CA. Proceedings, JPL Publication 97-21, p. 235-244.

KOKALY RF, DESPAIN DG, CLARK RN \& LIVO KE. 2003. Mapping vegetation in Yellowstone National Park using spectral feature analysis of AVIRIS data. Remote Sensing of Environment, 84: 437-456.

KRUSE FA, LEFKOFF AB, BOARDMAN JW, HEIEDBRECHT KB, SHAPIRO AT, BARLOON PJ \& GOETZ AFH. 1993. The Spectral Image Processing System (SIPS) - interactive visualization and analysis of imaging spectrometer data. Remote Sensing of Environment, 44: 145-163.

LICHTENTHALER HK. 1996. Vegetation stress: an introduction to the stress concept in plants. Journal of Plant Physiology, 148: 4-14.

MANTOVANI W \& MARTINS FR. 1988. Variações fenológicas das espécies do cerrado da Reserva biológica de Mogi-Guaçu, estado de São Paulo. Revista Brasileira de Botânica, 11: 101-112.

MORAN EF, BRONDIZIO E, MAUSEL P \& WU Y. 1994. Integrating Amazonian vegetation, land-use, and satellite data. BioScience. 44: 329-338. 
PONZONI FJ. 2001. Comportamento espectral da vegetação. In: P. R. MENESES; J. S. MADEIRA NETTO (Org.), Sensoriamento remoto - reflectância de alvos naturais. Brasília: Ed. UnB / Embrapa Cerrados. $262 p$.

QI J, CHEHBOUNI A, HUETE AR, KERR YH \& SOROOSHIAN S. 1994. A modified soil adjusted vegetation index. Remote Sensing of Environment, 48: 119-126.

RIBEIRO JF. 1998. Cerrado: matas de galeria. Planaltina: EMBRAPA -CPAC, $164 p$

RONDEAUX G, STEVEN M \& BARET F. 1996. Optimization of soiladjusted vegetation indices. Remote Sensing of Environment, 55: 95107.

ROUSE JW, HAAS RH, SCHELL JA, DEERING DW \& HARLAN JC. 1974. Monitoring the vernal advancement and retrogradation (greenwave effect) of natural vegetation. NASA/GSFC Final Report. Greenbelt, MD: NASA. $371 \mathrm{p}$.

ROWAN LC \& MARS JC. 2003. Lithologic mapping in the Mountain Pass, California area using Advanced Spaceborne Thermal Emission and Reflection Radiometer (ASTER) data. Remote Sensing of Environment, 84: 350-366.

SABOL DE, GILLESPIE AR, ADAMS JB, SMITH MO \& TUCKER CJ. 2002. Structural stage in Pacific Northwest forests estimated using simple mixing models of multispectral images. Remote Sensing of Environment, 80: $1-16$.

SARMIENTO G \& MONASTERIO M. 1983. Life forms and phenology.
In: BOULIERE F. (ed.). Determinants of tropical savannas. Paris: IUBS. p. $141-166$.

SOUZA GS. 1998. Introdução aos modelos de regressão linear e nãolinear, Brasília: EMBRAPA - SPI/EMBRAPA-SEA, 505 p.

STEEL RGD \& TORRIE JH. 1980. Principles and procedures of statistics: a biometrical approach. 2 ed. New York: McGraw-Hill. 631 p.

THOME K, ARAI K, HOOK S, KIEFFER H, LANG H, MATSUNAGA T, ONO A, PALLUCONI F, SAKUMA H, SLATER P, TAKASHIMA T, TONOOKA H, TSUCHIDA S, WELCH RM \& ZALEWSKI E. 1998. ASTER preflight and insight calibration and the validation of level 2 products. IEEE Transactions on Geoscience and Remote Sensing, 36(4): 1161-1172.

VAN LEEUWEN WJD \& HUETE AR. 1996. Effects of standing litter on the biophysical interpretation of plant canopies with spectral indices. Remote Sensing of Environment, 55: 123-134.

VIEIRA S. 1988. Indrodução a bioestatística. 5ed. Rio de Janeiro: Campus, $293 \mathrm{p}$.

WOOLEY JT. 1971. Reflectance and transmittance of light by leaves. Plant Physiology, 47: 656-662.

WOOLEY JT. 1973. Change of leaf dimensions and air volume with change in water content. Plant Physiology, 41: 815-816.

YAMAGUCHI Y, KAHLE AB, TSU H, KAWAKAMI T \& PNIEL M. 1998. Overview of Advanced Spaceborne Thermal Emission and Reflection Radiometer (ASTER). IEEE Transactions on Geoscience and Remote Sensing, 36(4): 1062-1071.

\section{NOTAS SOBRE OS AUTORES}

Osmar Abílio de Carvalho Júnior. Recebeu o título de geólogo pela Universidade de Brasília em 1990, e de mestre e doutor em prospecção mineral pela Universidade de Brasília em 1995 e 2000, respectivamente. Trabalhou como Pesquisador do Instituto Nacional de Pesquisas Espaciais (INPE) entre 2002 e 2004 . Atualmente é professor da Universidade de Brasília e bolsista de produtividade e pesquisa do CNPq onde realiza estudos sobre o processamento digital de imagens multiespectrais e hiperespectrais.

Renato Fontes Guimarães. Recebeu o título de engenheiro cartógrafo pela Universidade Estadual do Rio de Janeiro em 1987, o grau de mestre em geofísica pelo Observatório Nacional em 1991 e o grau de doutor em geologia pela Universidade Federal do Rio de Janeiro em 2000. Atualmente é professor Adjunto do Departamento de Geografia e chefe do Laboratório de Sistemas de Informações Espaciais do Departamento de Geografia. Possui como principais linhas de pesquisa o sensoriamento remoto e a modelagem matemática dos processos erosivos. É bolsista de produtividade e pesquisa do CNPq.

Éder de Souza Martins. Obteve a Graduação em Geologia pela Universidade de Brasília, UNB, em 1987. Realizou o mestrado em 1987 e doutorado 1999 ambos em Geologia pela Universidade de Brasília. Atualmente trabalha na Empresa Brasileira de Pesquisa Agropecuária, Centro de Pesquisa Agropecuária dos Cerrados, CPAC onde coordena o laboratório de pedologia. Realiza pesquisa em metodologias para o mapeamento e generalização cartográfica de informações pedológicas.

Ana Paula Ferreira de Carvalho. Recebeu o título de engenheira agronômica pela Universidade de Brasília em 1992, o grau de mestre em Ecologia pela Universidade de Brasília em 1998 e atualmente realiza o doutorado em bioquímica de plantas no Departamento de Ecologia da Universidade de Brasília. Trabalha na divisão de geoprocessamento do Instituto Nacional de Colonização e Reforma Agrária (INCRA).

Roberto Arnaldo Trancoso Gomes. Recebeu o título de geógrafo pela Universidade Federal do Rio de Janeiro em 1999, o grau de mestre em Geografia pela Universidade Federal do Rio de Janeiro em 2002 e atualmente realiza o doutorado utilizando modelagem matemática e sistema de informação geográfica no Departamento de Geografia da Universidade Federal do Rio de Janeiro. 